\title{
THE EXISTENCE OF SUPERSENSITIVE BOUNDARY-VALUE PROBLEMS
}

\begin{abstract}
Adriana Bohé
ABSTRACT. The phenomenon of supersensitivity in a general class of boundaryvalue problems $\varepsilon x^{\prime \prime}=f\left(t, x, x^{\prime}\right), 0<t<T, x(0)=A, x(T)=B$ is studied for a small positive $\varepsilon$. This appears as a consequence of the sensitive dependence of the shock location $t_{0}$ on the boundary conditions. Slight variations at the boundary values $A$ and $B$ rapidly change the position of $t_{0}$ of the jump in the interval $[0, T]$. In this work we show the relationship that exists between the supersensitivity of the boundary-value problem and the classical Rankine-Hugoniot shock condition. We give the geometrical features of the phenomenon, and we determine necessary and sufficient conditions on $f, A$, and $B$ for its occurrence. We also study the location of the shock as a function of the boundary data. We show that this extreme sensitivity to the boundary conditions is such that the transition between solutions with a boundary layer at 0 and those with a boundary layer at $T$ is continuous but very sharp. The phenomenon is illustrated with several examples and applications like the model of Burgers for turbulence or a model for compressible fluid flow in nozzles.
\end{abstract}

\section{Introduction}

Consider the singular boundary-value problem

$$
P(A, B) \quad\left\{\begin{array}{l}
\varepsilon x^{\prime \prime}=f\left(t, x, x^{\prime}\right) \\
x(0)=A, x(T)=B
\end{array} \quad 0<t<T\right.
$$

where $\epsilon$ is a fixed positive infinitesimal, $f$ is an internal smooth function, and $A$ and $B$ are real values.

This class of boundary-value problems arises in a variety of applied contexts such as fluid dynamics, chemical kinetics, and optimal control [3, 6, 7, 15, 16, 21, 22]. Applications of such two-point problems occur in the study of compressible fluids in gas dynamics [8], in studying exit time problems for stochastic differential equations [20], and in the study of contrast structures [5]. Other examples are given by the reactiondiffusion and phase transition models [13], the steady-state version of Burgers' model for turbulence [4], and problems related to critical paths of Feynman path integrals [19].

The solutions of such boundary-value problems have interesting features including boundary and interior layer regions. Determining the behavior of the solution presents both analytical and computational challenges because of these narrow regions where the solution changes rapidly.

Problems like $P(A, B)$ also involve other difficulties because of the phenomenon of supersensitivity exhibited by the solutions. This phenomenon appears as a consequence of the sensitive dependence of the shock location $t_{0}$ on the boundary conditions.

Received July 20, 1994, revised October 27, 1995.

1991 Mathematics Subject Classification: 34b15, 34d15, 34e15.

Key words and phrases: shock layers, singular perturbations, supersensitivity. 
Slight variations of the boundary values $A$ and $B$ may change rapidly the position $t_{0}$ of the jump in the interval $[0, T]$; see Figure 1.1.

The effect of these perturbations at the boundary conditions is such that the transition between solutions with a boundary layer at one endpoint of the interval $[0, T]$ and those with a boundary layer at the other endpoint is very sharp. More precisely, if a solution with an internal layer exists for the boundary conditions $\left(A^{*}, B^{*}\right)$, any other solution with an internal layer only may be obtained for values of $A$ and $B$ infinitely close to $A^{*}$ and $B^{*}$, respectively $\left(A \simeq A^{*}\right.$ and $B \simeq B^{*}$; i.e., in the halo of $A^{*}$ and $\left.B^{*}\right)$.

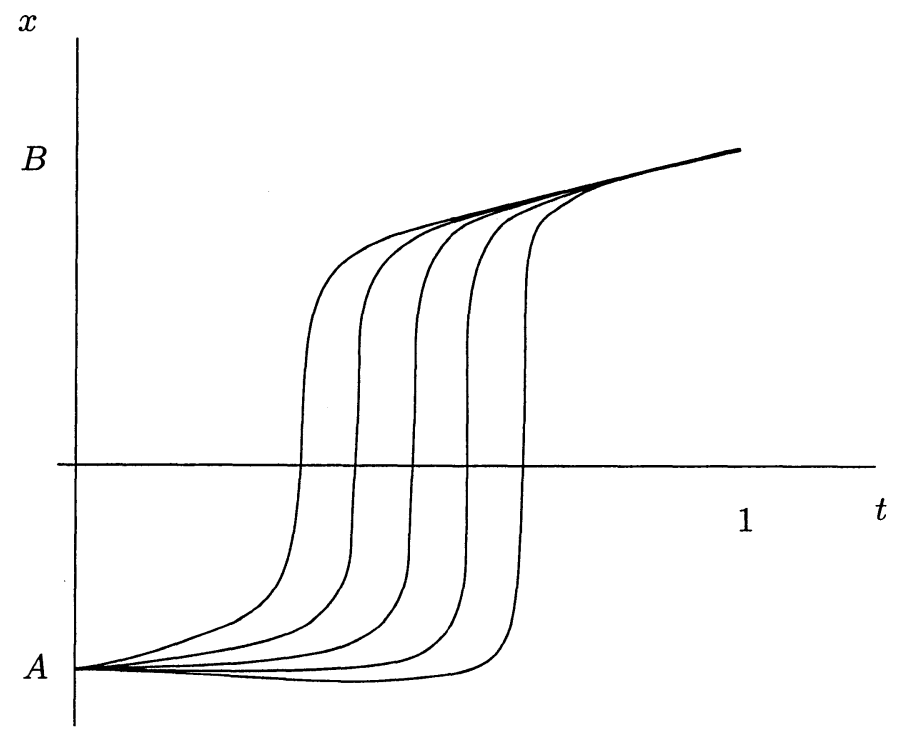

Figure 1.1. Solutions of $\varepsilon x^{\prime \prime}=-2 x x^{{ }^{[2]}}+2 t$ where $x^{{ }^{[2]}}=x^{\prime}\left|x^{\prime}\right|$ and $x(0)=A$ and $x(1)=B$ for five values of $A$ and $B$ close to -1 and $2^{2 / 3}$, respectively, and $\varepsilon=0.3$.

In [1], we have already studied this kind of supersensitive boundary-value problem for equations of the form $\varepsilon x^{\prime \prime}=g(x) f\left(x^{\prime}\right)$ with a Nagumo condition on $f$.

In this paper, our main objective is to study the phenomenon of supersensitivity in a general class of boundary-value problems $P(A, B)$ and to give necessary and sufficient conditions on $f, A$, and $B$ for the existence of such supersensitivity in $P(A, B)$. We use nonstandard analysis $[12,18]$. We adopt a geometrical approach to describe the solutions of $P(A, B)$ by examining the trajectories of the fast-slow system associated with (1) and to study the jumps of $P(A, B)$ by using the observability plane method [9].

We show the relationship that exists between the supersensitivity of $P(A, B)$ and the classical Rankine-Hugoniot shock condition in gas dynamics. We give the geometrical features of the phenomenon in the observability phase space, and we determine necessary and sufficient conditions for its occurrence. We also study the location of the shock as a function of the boundary conditions.

Finally, we illustrate this phenomenon with some examples and applications like the model of Burgers for turbulence and a model for compressible fluid flow in nozzles.

\section{Preliminaries}

In this section, we define the class of functions $f$ that we consider, and we give the definition of a supersensitive boundary-value problem. The nonstandard definitions 
of a jump, its thickness as well as its origin, and its extremity, used throughout this paper, correspond to those given in [1] and [10].

We consider the nonlinear singularly perturbed equation

$$
\varepsilon x^{\prime \prime}=f\left(t, x, x^{\prime}\right)
$$

in which $\partial f / \partial x^{\prime}=f_{x^{\prime}} \neq 0$ and $f$ has a type of growth with respect to $x^{\prime}$ defined as follows:

Definition 2.1. Let us consider a limited value $v_{0} \geq 0$ and an internal function $F:\left[v_{0},+\infty\right] \rightarrow \mathbb{R}^{+}, F$ of class $S^{0}$ and $C^{1}$, and such that the values of $F$ are not infinitesimal in $\left[v_{0},+\infty\right]$. We say that $F$ is a type of growth of $f$ for positive $v$ if there exists two internal functions $g(t, x)$ and $R(t, x, v)$, continuous and of class $S^{0}$, such that, for all limited values of $t$ and $x$ and for $v \geq v_{0}$,

$$
f(t, x, v)=g(t, x) F(v)+R(t, x, v)
$$

and

$$
R(t, x, v) / F(v) \simeq 0 \quad \text { for unlimited } v .
$$

The functions $g(t, x)$ and $R(t, x, v)$ are called the mantisse and the rest of $f$ with respect to $F$, respectively. We remark that if $F_{1}$ and $F_{2}$ are two types of growth of $f$, then there is a positive real value $K$ such that $F_{1}(v) / F_{2}(v) \simeq K$ for unlimited $v$.

A type of growth of $f$ for negative values of $v$ is defined analogously.

In particular, we consider functions $f$ having a type of growth $F$ such that

$$
\int_{v_{0}}^{\infty} v d v / F(v)=\infty
$$

This condition, which implies, in particular, the so-called Nagumo condition, restricts us to functions $f$ having at most a quadratic growth in $v$. We assume this hypothesis since solutions of equation (2) without such a growth restriction do not have jumps (see Vishik and Liusternik [25] or Diener [11]) except those called singular jumps. Readers may find a study of singular jumps of some singularly perturbed superquadratic equations like (2) in [2].

Let us consider the reduced problems

$$
\begin{array}{lll}
\left(P_{L}\right) & { }^{0} f\left(t, u, u^{\prime}\right)=0, & u(0)=A^{*}, \\
\left(P_{R}\right) & { }^{0} f\left(t, u, u^{\prime}\right)=0, & u(T)=B^{*}
\end{array}
$$

associated with the boundary-value problem $P(A, B)$, where $A^{*}={ }^{0} A$ and $B^{*}={ }^{0} B$.

Assume that there are functions $u_{L}$ and $u_{R}$ of class $C^{1}[0, T]$ satisfying $\left(P_{L}\right)$ and $\left(P_{R}\right)$, respectively, and such that $u_{L}$ does not intersect $u_{R}$ in $[0, T]$.

Definition 2.2. We say that $P(A, B)$ is a supersensitive boundary-value problem with respect to the boundary conditions $\left(A^{*}, B^{*}\right)$ if for any standard $t_{0} \in[0, T]$ there exists a pair of values $(A, B)$ with $A \simeq A^{*}$ and $B \simeq B^{*}$ such that the problem $P(A, B)$ has a solution $x(t)$ with a jump at $t_{0}$ and $x(t)$ satisfies

$$
x(t) \simeq\left\{\begin{array}{ll}
u_{L}(t) & 0 \leq t \leq t_{0}-\eta \\
u_{R}(t) & t_{0}+\delta \leq t \leq T
\end{array} \quad \text { for some } \eta \simeq 0^{+}, \delta \simeq 0^{+} .\right.
$$


An example of a supersensitive boundary-value problem is given by the two-point problem for Burgers' equation,

$$
\begin{aligned}
\varepsilon x^{\prime \prime} & =-x x^{\prime}, \\
x(0) & =A, \quad x(T)=B,
\end{aligned}
$$

with respect to the boundary conditions $A^{*}=-1$ and $B^{*}=1$.

Several studies $[6,14,21,23]$ concerning this problem describe the behavior of the solution as follows:

For $A=-1$ and any $B \gg 1$, the solution has a boundary layer at $t_{0}=0$ and $x(t) \simeq u_{L}(t)=-1$ for $0 \ll t \leq T$.

For $A=-1$ and for any $B$ such that $-1 \ll B \ll 1$, however, the solution exhibits a terminal layer at $t_{0}=T$ and $x(t) \simeq u_{R}(t)=1$ for $0 \leq t \ll T$.

Finally, for $A=-1$ and $B=1$, the solution involves an internal layer near $t_{0}=T / 2$ and $x(t)$ satisfies

$$
x(t) \simeq\left\{\begin{array}{ll}
-1 & 0 \leq t \leq t_{0}-\eta \\
1 & t_{0}+\eta \leq t \leq T
\end{array} \quad \text { where } \eta \simeq 0^{+} .\right.
$$

If we choose $B \simeq 1, B \neq 1$, however, we have shown [1] that the shock layer connecting the limiting solutions $u_{L}(t)=-1$ and $u_{R}(t)=1$ moves away from $t_{0}=T / 2$. More precisely, significant changes in the position of the shock layer are obtained by introducing exponentially small perturbations, $e^{-1 / b \varepsilon}$ for some limited positive $b$, of the boundary value $B^{*}=1(B \in \varepsilon$-microgal(1)). The solutions with a boundary layer at $t_{0}=0$ or at $t_{0}=T$ are obtained for the values of $B \simeq 1$ such that $B \notin \varepsilon$-microgal $(1)$; see Figure 2.1 .

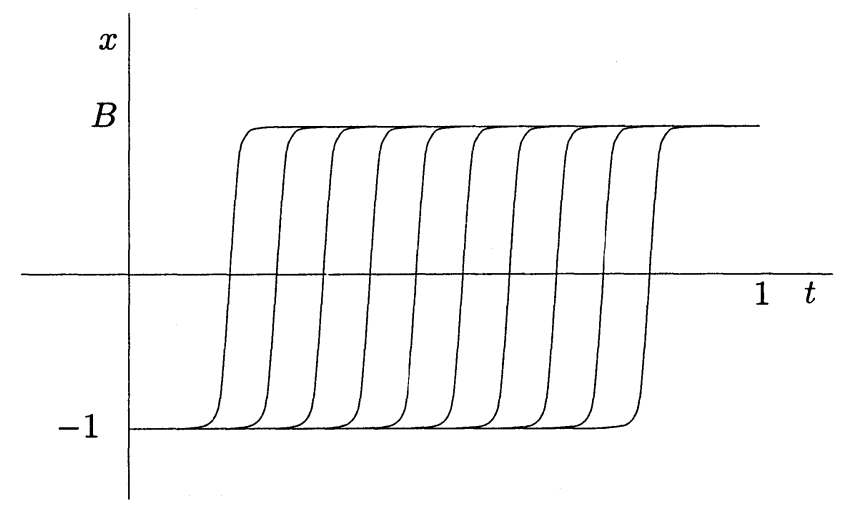

Figure 2.1. Solutions of $\varepsilon x^{\prime \prime}=-x x^{\prime}$, with $\varepsilon=0.05$ and $x(0)=-1$, $x(1)=B$, for nine values of $B$ close to 1 .

\section{The boundary-value problem $P(A, B)$ viewed in the observability space}

We shall adopt a geometric approach to describe the solutions of $P(A, B)$ by considering the differential equation (2) as the fast-slow system

$$
\begin{aligned}
t^{\prime} & =1, \\
x^{\prime} & =v, \\
\varepsilon v^{\prime} & =f(t, x, v),
\end{aligned}
$$


and examining the corresponding trajectories in the phase space $\left(t, x, v=x^{\prime}\right)$ and in the observability space $(t, x, V)$ defined below. A solution of $P(A, B)$ corresponds to a trajectory $\gamma(t)=(t, x(t), v(t))$ of $(7)$ which goes from some point in the vertical line $r_{A}$ defined by $t=0, x=A$ to the vertical line $r_{B}$ defined by $t=T, x=B$ in time $T$.

For limited values of $v=x^{\prime}$ the motion is rapid and nearly vertical, except near the slow surface $S$ defined by

$$
{ }^{0} f(t, x, v)=0 .
$$

The slow portions of the trajectory $\gamma(t)$ either are nearly vertical lines or lie near the surface $S$.

We call the region of $S$ where $\partial\left({ }^{0} f\right) / \partial v=\left({ }^{0} f\right)_{v}$ is negative "attractive" and the region where $\left({ }^{0} f\right)_{v}$ is positive "repulsive". We denote those regions $S_{A}$ and $S_{R}$, respectively.

For unlimited values of $v$, the rapid portions of $\gamma(t)$ which correspond to the jumps of the solution $x(t)$ of (2) may be examined by means of the observability method [9]. This method consists in rescaling the fast variable $v$ by introducing $v=h(V / \varepsilon)$ where $h$ is defined by

$$
\begin{aligned}
h h^{\prime} & =F(h), \\
h(0) & =v_{0} \quad \text { with } v_{0} \text { a limited value. }
\end{aligned}
$$

The function $h$ depends on the type of growth $F$ of $f$ and is such that the new variable $V$ remains limited during the jump. After performing the change of variable, the rapid trajectories satisfy

$$
\begin{aligned}
\frac{d t}{d x} & =\frac{1}{h}, \\
\frac{d V}{d x} & =g(t, x)+\frac{R(t, x, h)}{F(h)} .
\end{aligned}
$$

Because of the properties of $f(t, x, v)$ for unlimited $v$, this system is infinitely close to

$$
\begin{aligned}
\frac{d t}{d x} & =0 \\
\frac{d V}{d x} & ={ }^{0} g(t, x),
\end{aligned}
$$

so any jump at $t=t_{0}$ is contained in the halo of the vertical plane $t=t_{0}$ and is infinitely close to the curve of the equation

$$
V(x)=\int_{x_{0}}^{x}{ }^{0} g\left(t_{0}, s\right) d s+V\left(x_{0}\right),
$$

as was shown by Diener $\left[10\right.$, Thm.1, p. 545]. The plane $t=t_{0}$ which contains the shadows of the jumps at $t=t_{0}$ in the $(x, V)$ plane is called the observability plane of the jumps. The rapid motions of any trajectory related to a jump at $t_{0}$ move, in the $(x, V)$ plane $t=t_{0}$, from the left to the right in the positive half-plane (increasing jumps) and from the right to the left in the negative portion (decreasing jumps).

Remark 3.0.1. For the equation $\varepsilon x^{\prime \prime}=-2 x x^{\prime}+1-x^{2}$, a type of growth for both positive and negative $v$ is given by $F(v)=v$ while the mantisse is $g(t, x)=-2 x$. Then, the increasing and the decreasing jumps of the solutions of this equation at $t=t_{0}$ are both infinitely close to the parabolas $V=-x^{2}+C, C$ a constant, in the $(x, V)$ plane. In Figure 3.1, we have drawn three parabolas. The portions of these curves which are contained in the positive half-plane correspond to the increasing jumps while those portions which are contained in the region $V<0$ correspond to the decreasing jumps. 
The arrows on the parabolas show the direction in which the point $(x, V(x))$ describes each trajectory.

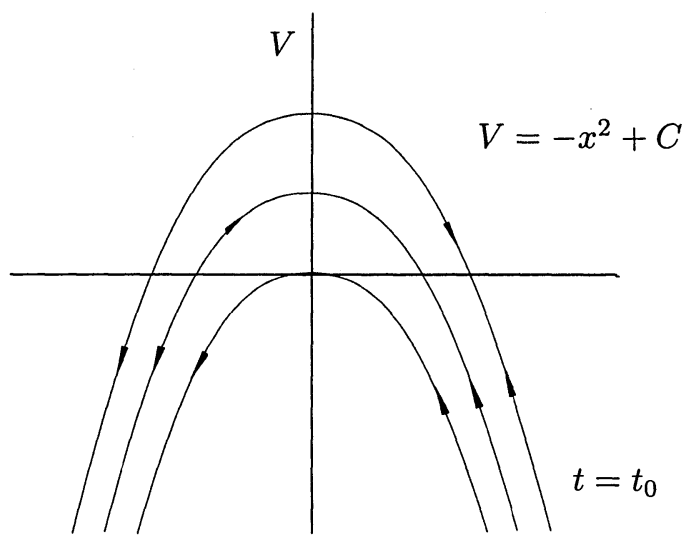

Figure 3.1. The jumps of the solutions of $\varepsilon x^{\prime \prime}=-2 x x^{\prime}+1-x^{2}$ in the observability plane $t=t_{0}$.

The slow motions (for limited $v$ ) of the trajectory $\gamma(t)$, which satisfies ${ }^{0} f(\gamma(t))=0$ in the phase space $(t, x, v)$, appear in the observability space $(t, x, V)$ near the horizontal plane defined by $V=0$.

Typical trajectory associated with a solution $x(t)$ of problem $P(A, B)$ with a jump at some $t=t_{0} \in(0, T)$ and such that $x(t)$ satisfies (6) is sketched in Figure 3.2.

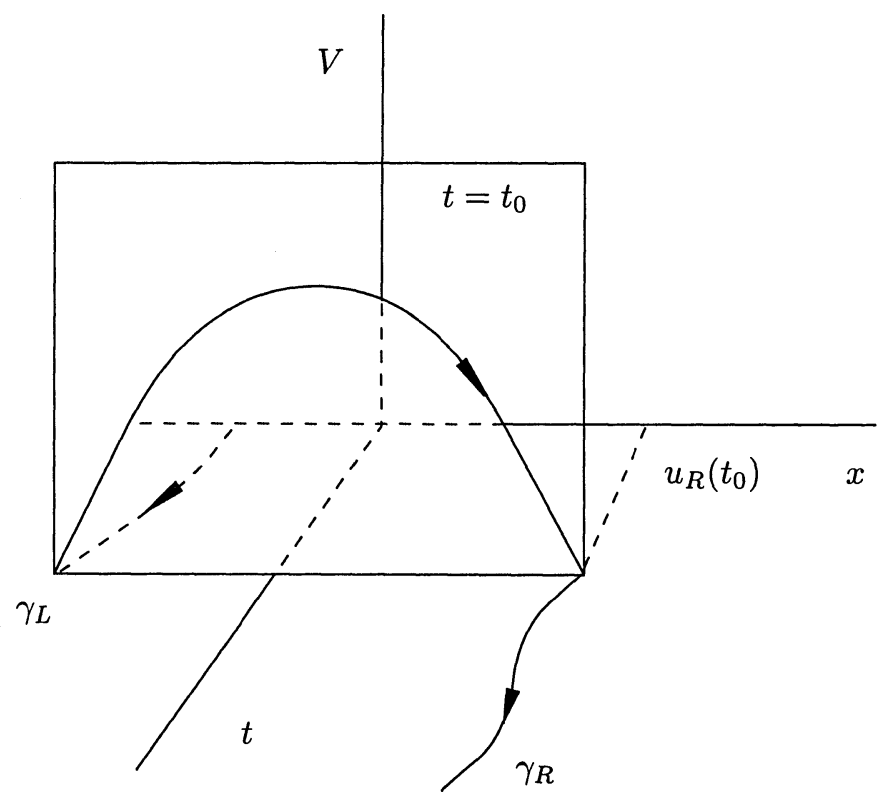

FIgURE 3.2. The shadows $\gamma_{R}(t)=\left(t, u_{R}, u_{R}^{\prime}\right)$ and $\gamma_{L}(t)=\left(t, u_{L}, u_{L}^{\prime}\right)$ of the slow motions of $\gamma(t)$ are contained, in the observability space, in the horizontal plane $V=0$. The jump is contained in the vertical plane $t=t_{0}$. 
In order to complete the description of the trajectory $\gamma(t)$ associated with a solution $x(t)$ of $P(A, B)$ in the observability space, we shall need to make precise the behavior of the jump of $x(t)$ near $V=0$.

Suppose a solution $x(t)$ has a jump at $t=t_{0}$ and the jump has an extremity $x_{+}$. Roughly speaking this means that $\gamma(t)$ has finished jumping at the value $x_{+}$for $t$ near $t_{0}$ and then it continues as a slow trajectory.

Then, Theorem $2[10$, p. 548] states that the shadow of the jump must satisfy $V\left(x_{+}\right)=0$ in the $(x, V)$ plane. Analogously, if the jump has an origin $x_{-}$, then the shadow of the corresponding trajectory in the observability plane satisfies $V\left(x_{-}\right)=0$. Therefore, any jump at $t=t_{0}$ having both an origin $x_{-}$and an extremity $x_{+}$satisfies

$$
\int_{x_{-}}^{x_{+}} 0\left(t_{0}, s\right) d s=0,
$$

which is nothing more than the Rankine-Hugoniot condition.

Conversely, if there is a value $x_{+}>x_{0}$ such that the shadow of the jump satisfies $V\left(x_{+}\right)=0$, and if $V(x) \neq 0$ for all $x \in\left[x_{0}, x_{+}\right]$and $V^{\prime}\left(x_{+}\right)={ }^{0} g\left(t_{0}, x_{+}\right) \neq 0$, then $x_{+}$ is the extremity of the jump.

Analogously, if there is a value $x_{-}<x_{0}$ such that the shadow of the jump satisfies $V\left(x_{-}\right)=0$ and if $V(x) \neq 0$ for all $x \in\left[x_{-}, x_{0}\right]$ and $V^{\prime}\left(x_{-}\right)={ }^{0} g\left(t_{0}, x_{-}\right) \neq 0$, then $x_{-}$ is the origin of the jump.

\section{Necessary and sufficient conditions for a supersensitive boundary-value problem}

4.1. Geometrical features. In this section, we show the relationship between a supersensitive two-point problem and the Rankine-Hugoniot condition and give a geometric interpretation of this condition.

Assume:

$\left.\mathrm{H}_{1}\right) f(t, x, v)$ is an internal smooth function with $F(v)$ as a type of growth of $f$ for both positive and negative $v$, and the functions $g(t, x)$ and $R(t, x, v)$ are the mantisse and the rest, respectively.

$\mathrm{H}_{2}$ ) $F$ satisfies the condition (3).

$\left.\mathrm{H}_{3}\right)$ there are functions $u_{L}$ and $u_{R}$ of class $C^{1}[0, T]$ satisfying $\left(P_{L}\right)$ and $\left(P_{R}\right)$, respectively, and such that $u_{L}(t)-u_{R}(t) \neq 0$ for all $t \in[0, T]$. (We henceforth shall suppose that $u_{L}(t)<u_{R}(t)$.)

Let us consider the function $J:[0, T] \rightarrow \mathbb{R}$ defined by

$$
J(t)=\int_{u_{L}(t)}^{u_{R}(t)} 0 g(t, s) d s .
$$

Theorem 4.1.1. Consider the boundary-value problem $P(A, B)$, and suppose hypotheses $\mathrm{H}_{1}, \mathrm{H}_{2}$, and $\mathrm{H}_{3}$ are satisfied. If $P(A, B)$ is supersensitive with respect to $\left(A^{*}, B^{*}\right)$, then $J(t) \equiv 0$ in $[0, T]$.

Proof. For any standard value $t_{0} \in(0, T)$, there are $A \simeq A^{*}$ and $B \simeq B^{*}$ such that $P(A, B)$ has a solution $x(t)$ with a jump at $t=t_{0}$, and $x(t)$ satisfies $(6)$ since $P(A, B)$ is assumed to be supersensitive. Therefore, for any standard $t_{0} \in(0, T)$, the jump is an internal layer having $x_{-}=u_{L}\left(t_{0}\right)$ as the origin and $x_{+}=u_{R}\left(t_{0}\right)$ as the extremity. Then, it follows from Theorem $2\left[10\right.$, p. 548] that $J\left(t_{0}\right)=0$ for any standard $t_{0} \in(0, T)$ and, since $J(t)$ is a standard continuous function, we have $J(t) \equiv 0$ for all $t \in[0, T]$. 
Remark 4.1.2. Theorem 4.1.1. gives a necessary condition for the existence of the phenomenon of supersensitivity. The boundary-value problem must satisfy the Rankine-Hugoniot condition in the whole interval $[0, T]$. We can interpret this result in the observability space $(t, x, V)$ as follows.

Let us consider the surface $S^{*}$ defined by

$$
S^{*}=\left\{(t, x, V): t \in[0, T], \quad u_{L}(t) \leq x \leq u_{R}(t), V(t, x)=G(t, x)\right\}
$$

where the function $G:[0, T] \times \mathbb{R} \rightarrow \mathbb{R}$ is defined by

$$
G(t, x)=\int_{u_{L}(t)}^{x}{ }^{0} g(t, s) d s .
$$

This surface is built up by considering any possible jump in $[0, T]$ from the slow solution $x(t)=u_{L}(t)$.

When $P(A, B)$ is supersensitive, for any standard $t_{0} \in[0, T]$, there is a trajectory $\gamma(t)$ that remains, in the $(t, x, V)$ space, in the halo of $V=0$ with $x(t) \simeq u_{L}(t)$ for $0 \leq t<t_{0}$ and with $x(t) \simeq u_{R}(t)$ for $T \geq t>t_{0}$, while the portion of $\gamma(t)$ associated with the jump remains infinitely close to the surface $S^{*}$. That is, the two "equilibrium states" $u_{L}(t)$ and $u_{R}(t)$ may be connected with a shock at every $t_{0} \in[0, T]$. Then, in terms of the geometry of the surface $S^{*}$, Theorem 4.1 .1 says that $S^{*}$ also must intersect the plane $V=0$ at the other reduced solution $u_{R}(t)$ in the whole interval $[0, T]$.

Let us now investigate the existence of supersensitive boundary layers and free layers by assuming the following hypothesis $\mathrm{H}$ :

$\mathrm{H}_{1}$ ) and $\mathrm{H}_{2}$ ),

$\left.\mathrm{H}_{3}^{\prime}\right)$ the reduced problem ${ }^{0} f\left(t, u, u^{\prime}\right)=0$ has a unique solution $u \in C^{1}[0, T]$ in $([0, T] \times \mathbb{R})$,

$\mathrm{H}_{4}$ ) the boundary conditions $A^{*}$ and $B^{*}$ are such that the reduced solutions $u_{L}$ and $u_{R}$ of $\left(P_{L}\right)$ and $\left(P_{R}\right)$, respectively, satisfy $J(t) \equiv 0$ in $[0, T]$,

$\left.\mathrm{H}_{5}\right)$ any reduced solution $u(t)$ satisfies in $[0, T]$,

$$
\begin{array}{ll}
\left(t, u, u^{\prime}\right) \in S_{A}, & \text { if } u(t) \geq u_{R}(t), \\
\left(t, u, u^{\prime}\right) \in S_{R}, & \text { if } u(t) \leq u_{L}(t),
\end{array}
$$

$\left.\mathrm{H}_{6}\right) G(t, x) \gg 0$ for all $0 \leq t \leq T$ and $u_{L}(t) \ll x \ll u_{R}(t)$,

$\left.\mathrm{H}_{7}\right)$

$$
\begin{array}{ll}
{ }^{0} g(t, x) \ll 0, & \text { if } x \geq u_{R}(t), \\
{ }^{0} g(t, x) \gg 0, & \text { if } x \leq u_{L}(t), \text { for } 0 \leq t \leq T .
\end{array}
$$

Remark 4.1.3. In condition $\left.\mathrm{H}_{5}\right)$, we assume that the corresponding trajectory $(t, u(t)$, $\left.u^{\prime}(t)\right)$ is contained either in the attractive region $S_{A}$ of the slow surface $S$ if $u(t) \geq$ $u_{R}(t)$ for $t \in[0, T]$ or in the repulsive region $S_{R}$ of $S$ if $u(t) \leq u_{L}(t)$.

The conditions $\mathrm{H}_{6}$ ) and $\mathrm{H}_{7}$ ) are nothing more than geometric restrictions on the surface $S^{*}$ of the jumps. If the reduced solutions are such that $u_{L}(t)>u_{R}(t)$, we will require in $\left.\mathrm{H}_{6}\right)$ that $G(t, x) \ll 0$ for all $0 \leq t \leq T$ and $u_{L}(t) \gg x \gg u_{R}(t)$ and in $\left.\mathrm{H}_{7}\right)$ that ${ }^{0} g(t, x) \ll 0$ if $x \leq u_{R}(t)$ and ${ }^{0} g(t, x) \gg 0$ if $x \geq u_{L}(t)$, for $0 \leq t \leq T$. 
Let us assume $A$ is fixed, $A=A^{*}, B$ is a limited value where ${ }^{0} B$ is not necessarily equal to $B^{*}$, and $u(t)$ is the solution of the reduced problem

$$
{ }^{0} f\left(t, u, u^{\prime}\right)=0, \quad u(T)={ }^{0} B .
$$

We recall that $u_{R}(t)$ denotes the solution of $(16)$ if ${ }^{0} B=B^{*}$. Because of the uniqueness of solution of the reduced problem $u(t) \equiv u_{R}(t)$ if $B \simeq B^{*}$. For $B \not B^{*}$, any reduced solution $u(t)$ satisfies in $[0, T]$ either $u_{L}(t)<u(t)<u_{R}(t)$ if $B_{L} \ll B \ll B^{*}$ or $u(t)>u_{R}(t)$ if $B \gg B^{*}$ where we note $B_{L}=u_{L}(T)$ with $u_{L}(t)$ the solution of the reduced problem (4).

Lemma 4.1.4. Assume hypothesis $\mathrm{H}$ is satisfied. Then, for any $B \gg B_{L}, B$ limited, $P(A, B)$ has a solution $x(t)$ with a jump at some $t_{0} \in[0, T]$ and

$$
x(t) \simeq\left\{\begin{array}{ll}
u_{L}(t), \quad 0 \leq t \leq t_{0}-\eta \\
u_{R}(t), & t_{0}+\delta \leq t \leq T
\end{array} \quad \text { for some } \eta \simeq 0^{+}, \delta \simeq 0^{+} .\right.
$$

In addition,

(1) if $B \gg B^{*}, x(t)$ has a boundary layer at $t_{0}=0, x^{\prime}(0) \simeq+\infty$ and $x(t) \simeq u(t)$ in $[\delta, T], \delta \simeq 0^{+}$.

(2) if $B_{L} \ll B \ll B^{*}, x(t)$ has a boundary layer $t_{0}=T, x^{\prime}(T) \simeq+\infty$ and $x(t) \simeq u_{L}(t)$ in $[0, T-\eta], \eta \simeq 0^{+}$.

Proof. For $B$ limited and $B \gg B_{L}$, we note $V_{B}=-G(0, u(0))$ where $u(t)$ is the solution of (16). We remark that $V_{B}<0$ if $B_{L} \ll B \ll B^{*}, V_{B}=0$ if $B \simeq B^{*}$ (in this case $\left.u(0)=u_{R}(0)\right)$, and $V_{B}>0$ if $B \gg B^{*}$ in virtue of the hypothesis H. Let us consider, in the $(t, x, V)$ space, the trajectory $\gamma(t)$ of $(7)$ starting at a point $\left(0, A, V_{0}\right)$, $A=A^{*}$ in the vertical line $r_{A}$.

If $V_{0} \gg \max \left\{0, V_{B}\right\}$, (see Fig.4.1 (a) and Fig.4.2 (a)) $\gamma(t)$ remains, in the plane $t=0$, infinitely close to the curve of equation

$$
V=G(0, x)+V_{0}
$$

until it reaches the halo of $V=0$ near the point $\left(0, A_{1}, 0\right)$ where $A_{1}$ is such that $G\left(0, A_{1}\right)+V_{0}=0$ and $A_{1} \gg u(0)$. As $(\partial V / \partial x)\left(0, A_{1}\right)={ }^{0} g\left(0, A_{1}\right) \ll 0$, Theorem 2 [10, p. 548] ensures that $A_{1}$ is the extremity of the jump and the trajectory reaches the halo of the slow surface $S$. Then, as a consequence of $\mathrm{H}_{5}$ ), it continues infinitely close to the slow solution $\left(t, u_{1}(t), u_{1}^{\prime}(t)\right)$ where $u_{1}(0)=A_{1} \gg u(0)$ in $[0, T]$. Then $x(T) \simeq u_{1}(T)>B$.

If $V_{0} \ll \max \left\{0, V_{B}\right\}$ and $V_{0}<0$, (see Fig.4.1 (b)) $\gamma(t)$ remains infinitely close to the curve of equation (17) up to an unlimited negative value of $x$ since $G(0, x)$ is an increasing function of $x$ for $x \leq A$, and then $\gamma(t)$ moves out of the limited space $(t, x, V)$, and hence $x(T)<B$. On the contrary, if $V_{0} \geq 0$ (Fig.4.2 (b)), $\gamma(t)$ satisfies (up to an infinitesimal) the curve of equation (17) until it reaches the halo of $V=0$ near the point $\left(0, A_{2}, 0\right)$ where $A_{2}$ is such that $G\left(0, A_{2}\right)+V_{0}=0$ and $u_{R}(0) \leq A_{2} \ll$ $u(0)$. Then, it continues infinitely close to the slow solution $\left(t, u_{2}(t), u_{2}^{\prime}(t)\right)$ where $u_{2}(0)=A_{2}$, and finally, $x(T)<B$.

By continuity of the flow, there is $V_{0} \simeq \max \left\{0, V_{B}\right\}$ for which $x(T)=B$. This proves the existence of a solution of $P(A, B)$ with a jump at some $t_{0}$ in $[0, T]$ and satisfying the prescribed behavior.

In addition, if $B \gg B^{*}, \max \left\{0, V_{B}\right\}=V_{B}$. Then $x(t)$ has a boundary layer at $t_{0}=0, x^{\prime}(0) \simeq+\infty$ and $x(t) \simeq u(t)$ in $\left[\delta^{+}, T\right], \delta \simeq 0^{+}$, which proves (1). If $B_{L} \ll B \ll B^{*}, \max \left\{0, V_{B}\right\}=0$, and $x(t)$ has a boundary layer $t_{0}=T$. In fact, 
if we suppose that the solution $x(t)$ jumps at some $t_{0} \neq T$, then the corresponding trajectory $\gamma(t)$ would remain in the halo of $V=0$ for $0 \leq t \leq t_{1}$ where $t_{1} \simeq t_{0}$ while the solution would satisfy $x(t) \simeq u_{L}(t)$ in $\left[0, t_{1}\right]$. Then $\gamma(t)$ would reach the halo of $V=0$ at some $t_{2} \simeq t_{0}, x\left(t_{2}\right) \simeq u_{R}\left(t_{0}\right)$ where $(\partial V / \partial x)\left(t_{0}, u_{R}\left(t_{0}\right)\right) \ll 0$. Finally, $\gamma(t)$ would lie near the plane $V=0$ with $x(t) \simeq u_{R}(t)$ up to time $T$, and then it would never be able to reach the vertical line $r_{B}$, which is absurd for a solution of $P(A, B)$. Therefore, in order to join $r_{A}$ with $r_{B}, \gamma(t)$ must remain in the halo of $V=0$ with $x(t) \simeq u_{L}(t)$ and must reach $r_{B}$ with a jump in the halo of $t_{0}=T$, which proves (2).

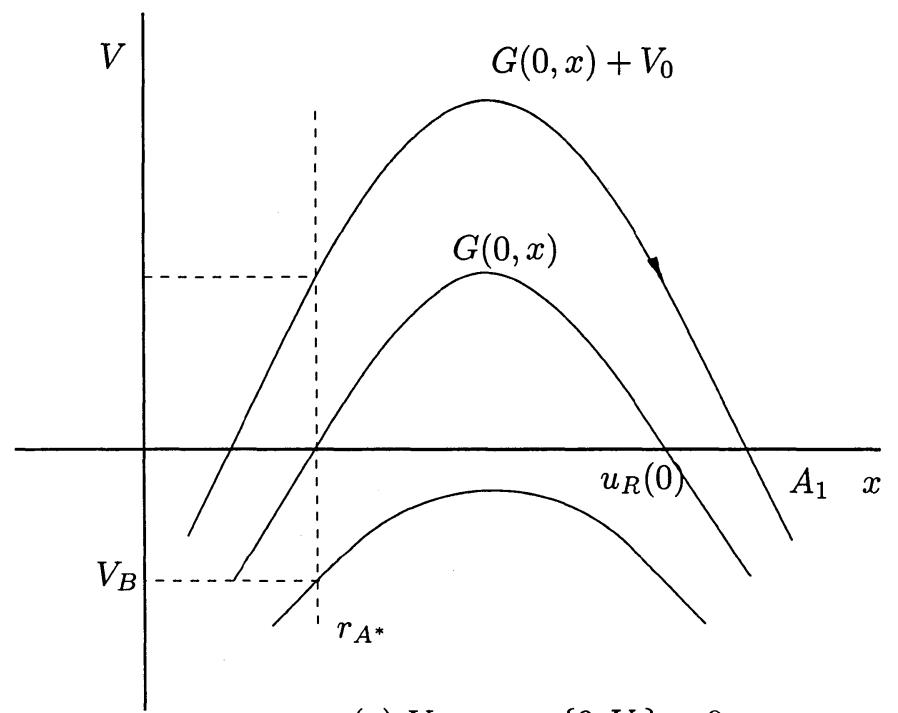

(a) $V_{0} \gg \max \left\{0, V_{b}\right\}=0$

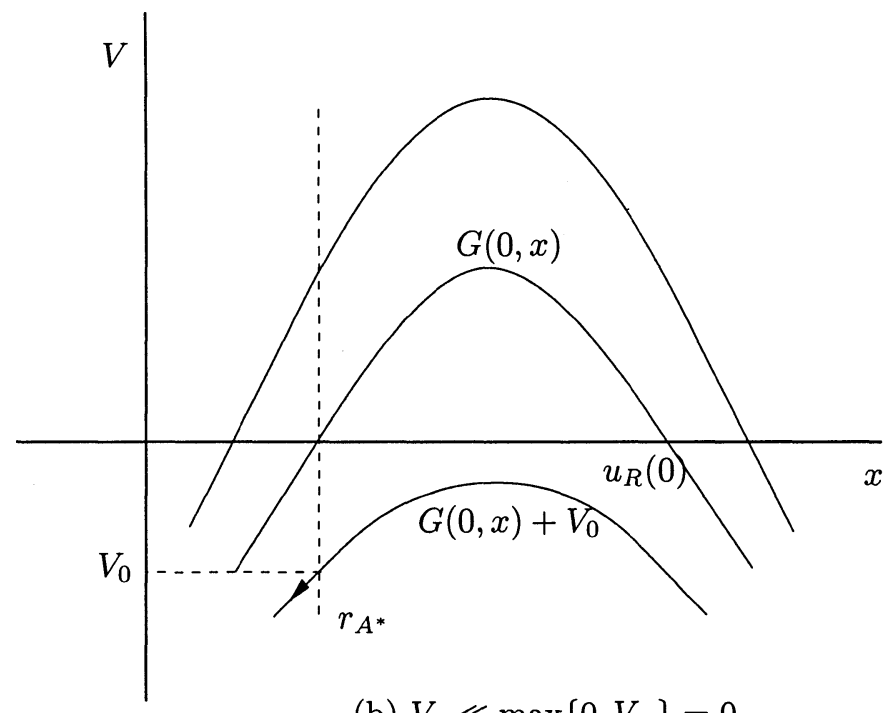

(b) $V_{0} \ll \max \left\{0, V_{B}\right\}=0$

Figure 4.1. The trajectory $\gamma(t)$ in the observability plane $t=0$ for the case $B_{L} \ll B \ll B^{*}$. 


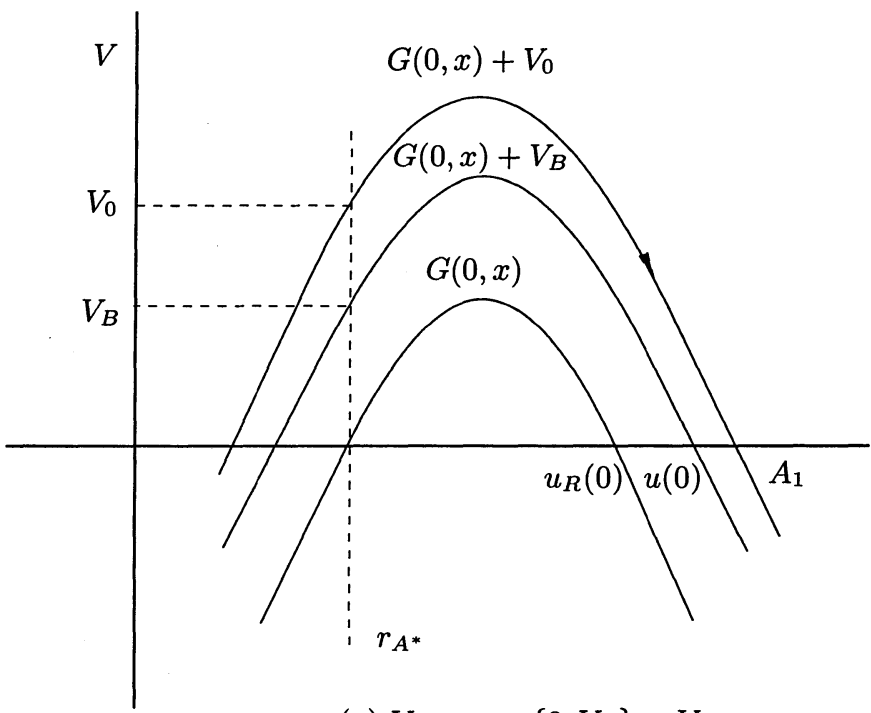

(a) $V_{0} \gg \max \left\{0, V_{B}\right\}=V_{B}$

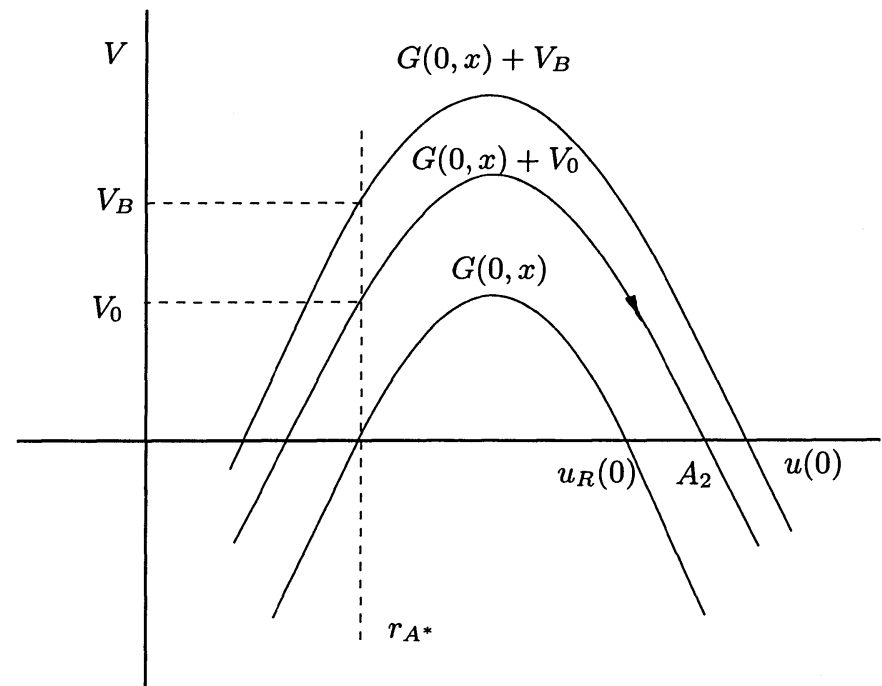

(b) $V_{0} \ll \max \left\{0, V_{B}\right\}=V_{B}$

Figure 4.2. The trajectory $\gamma(t)$ in the observability plane $t=0$ for the case $B \gg B^{*}$.

Remark 4.1.5. When $B \simeq B^{*}, \max \left\{0, V_{B}\right\}=0$, but any $t_{0} \in[0, T]$ will allow $\gamma(t)$ to join $r_{A}$ with $r_{B}$. We now will show that $P(A, B)$ also has solutions with a boundary layer at one endpoint and at the other for values of $B \simeq B^{*}$.

Lemma 4.1.6. Assume the hypothesis $H$. Then, there are $B_{1}<B^{*}$ and $B_{2}>B^{*}$, $B_{1} \simeq B^{*} \simeq B_{2}$, such that $P(A, B)$ has a solution with a boundary layer at $t_{0}=0$ if $B=B_{2}$ or at $t_{0}=T$ if $B=B_{1}$.

Proof. Let us consider $\mathbf{H}_{1}=\left\{B \mid \exists x\left[(x(t) \in \Omega)\right.\right.$ and $\left(\exists \eta \simeq 0^{+} \forall t \in[0, T-\eta], x(t) \simeq\right.$ $\left.u_{L}(t)\right)$ and $\left.\left.\left(x^{\prime}(T) \simeq+\infty\right)\right]\right\}, \Omega=\{x(t) \mid x(t)$ is a solution of $P(A, B)\}$ and 
$\mathbf{G}_{1}=\left\{B: B_{L} \ll B \ll B^{*}\right\} . \mathbf{H}_{1}$ is a prehalo since we have

$$
\mathbf{H}_{1}=\bigcap_{\varepsilon, \mu>0} H_{\varepsilon, \mu, n>0} s t
$$

where $H_{\varepsilon, \mu, n}=C_{\varepsilon, \mu} \bigcap D_{n}$,

$$
C_{\varepsilon, \mu}=\left\{B \mid \exists x\left[(x(t) \in \Omega) \text { and }\left(\exists \eta<\mu \forall t \in[0, T-\eta]\left|x(t)-u_{L}(t)\right|<\varepsilon\right)\right]\right\},
$$

and $D_{n}=\left\{B \mid \exists x\left[(x(t) \in \Omega)\right.\right.$ and $\left.\left.\left(x^{\prime}(T)>n\right)\right]\right\}$. It is easy to show that $\mathbf{G}_{1}$ is a galaxy. By Lemma 4.1.4, this external set is included in $\mathbf{H}_{1}$. By the Permanence Principle, this inclusion is strict (cf. [12] and [24]). Therefore, there is $B_{1}<B^{*}$, with $B_{1} \simeq B^{*}$ such that $P\left(A, B_{1}\right)$ has a solution $x(t)$ with a boundary layer at $t_{0}=T$ and $x(t) \simeq u_{L}(t)$ in $[0, T-\eta], \eta \simeq 0^{+}$. The existence of $B_{2}>B^{*}, B_{2} \simeq B^{*}$, for which $P\left(A, B_{2}\right)$ has a solution with a boundary layer at $t=0$ and $x(t) \simeq u_{R}(t)$ in $[\eta, T]$, $\eta \simeq 0^{+}$follows analogously by considering $\mathbf{G}_{2}=\left\{B: B \ll B^{*}\right\}$ and

$$
\mathbf{H}_{1}=\left\{B \mid \exists x\left[x(t) \in \Omega \text { and }\left(\exists \delta \simeq 0^{+} \forall t \in[\delta, T] x(t) \simeq u(t)\right) \text { and }\left(x^{\prime}(0) \simeq+\infty\right)\right]\right\}
$$

where $u(t)$ is the solution of (16).

\subsection{Existence of supersensitivity and continuous dependence on the bound- ary conditions. Let us fix $A=A^{*}$.}

Theorem 4.2.1. Assume the hypothesis $\mathrm{H}$ is satisfied. Then, $P(A, B)$ is a supersensitive boundary-value problem with respect to $\left(A^{*}, B^{*}\right)$.

Proof. For any standard $t_{0} \in(0, T)$, let us consider the boundary-value problem $\varepsilon x^{\prime \prime}=$ $f\left(t, x, x^{\prime}\right), x(0)=A, x\left(t_{0}\right)=M$ where $M=\left(u_{L}\left(t_{0}\right)+u_{R}\left(t_{0}\right)\right) / 2$. Arguing as in Lemma 4.1.4, we see that this problem has a solution $x(t)$ with a boundary layer at the endpoint $t_{0}$ and $x(t) \simeq u_{L}(t)$ for $0 \leq t \leq t_{0}-\eta$, with $\eta \simeq 0^{+}$. This jump has $x_{-}=u_{L}\left(t_{0}\right)$ as the origin, and it is infinitely close to the curve of the equation $V=G\left(t_{0}, x\right)$, in the $(t, x, V)$ space. It follows from the Short Shadow Lemma [12] that the trajectory $\gamma(t)$ associated with $x(t)$ extends throughout $[0, T]$, it satisfies $V \simeq G\left(t_{0}, x\right)$ up to $x \simeq u_{R}\left(t_{0}\right)$ where $G\left(t_{0}, x\right)$ vanishes, and then, in the $(t, x, v)$ space, $\gamma(t)$ continues infinitely close to the attractive slow solution $\left(t, u_{R}(t), u_{R}^{\prime}(t)\right)$ up to $t=T$ where $x(T) \simeq B^{*}$. Then, for any standard $t_{0} \in(0, T)$, there exist $B=x(T) \simeq B^{*}$ such that the solution $x(t)$ of $P(A, B)$ has a jump at $t_{0}$ and satisfies (6). For $t_{0}=0$ and $t_{0}=T$, the existence of $B \simeq B^{*}$ such that $P(A, B)$ has a solution with a jump at $t_{0}$ and satisfies (6) is given by Lemma 4.1 .6 , and this concludes the proof.

Now we are interested in studying how the shock location depends on the boundary conditions.

Let us consider $B_{1}$ and $B_{2}$ the values given by Lemma 4.1.6. We denote by $t_{B}$ the value of $t$ such that a solution $x(t)$ of $P\left(A^{*}, B\right)$ satisfies

$$
x\left(t_{B}\right)=\frac{\left(u_{L}\left(t_{B}\right)+u_{R}\left(t_{B}\right)\right)}{2} .
$$

Then $t_{B_{1}} \simeq T$ and $t_{B_{2}} \simeq 0^{+}$.

We define the function $\phi:\left[B_{1}, B_{2}\right] \rightarrow\left[t_{B_{2}}, t_{B_{1}}\right]$ by $\phi(B)=t_{B}$.

Theorem 4.2.2. Suppose the hypothesis $\mathrm{H}$ is satisfied. If the solutions of boundaryvalue problems for $\varepsilon x^{\prime \prime}=f\left(t, x, x^{\prime}\right)$ on each subinterval $I \subseteq[0, T]$ are unique, then $\phi$ is a decreasing continuous function on each $\left[B_{1}, B\right] \subset\left[B_{1}, B_{2}\right]$ where ${ }^{0}\left(t_{B}\right)>0$. In addition, if $u_{L}(t)+u_{R}(t)$ decreases at 0 , then $\phi$ is continuous on $\left[B_{1}, B_{2}\right]$. 
Proof. For any $B \in\left[B_{1}, B_{2}\right]$, Lemma 4.1.4 insures that $P\left(A^{*}, B\right)$ has a solution $x(t)$ with an increasing jump at some standard $t_{0} \in[0, T]$. Since $x(t)$ satisfies $(6)$ and is unique, there is a unique $t_{B}$ such that ${ }^{0}\left(t_{B}\right)=t_{0}$ and $\phi(B)=t_{B}$. Then $\phi$ is welldefined, and $\phi$ is a decreasing function because of the uniqueness of solutions of (2) on each $[0, t] \subseteq[0, T]$.

Let us show that $\phi$ is a surjection in any subinterval $\left[B_{1}, B\right], B<B_{2}$ where $B$ is such that $t_{B}=\phi(B) \gg 0$. In fact, for any $\tau \in\left[t_{B_{1}}, t_{B_{2}}\right]$ with $\tau \gg 0$, we prove as in Theorem 4.2.1 that the boundary-value problem $P_{M}: \varepsilon x^{\prime \prime}=f\left(t, x, x^{\prime}\right), x(0)=A$, $x(\tau)=M$ with $M=\left(u_{L}(\tau)+u_{R}(\tau)\right) / 2$, has a unique solution $x(t)$ with a boundary layer at the "endpoint" $\tau$. The solution extends throughout $[0, T]$, and it satisfies $x(T)=B \simeq B^{*}$, where $B$ must belong to $\left[B_{1}, B_{2}\right)$ by virtue of the uniqueness of solutions of boundary-value problems. Then, for any $\tau \in\left[t_{B_{2}}, t_{B_{1}}\right]$ with $\tau \gg 0$, there is $B \in\left[B_{1}, B_{2}\right)$ such that $\phi(B)=\tau$. Thus, $\phi$ is a surjection in each $\left[\tau, t_{B_{1}}\right]$ where $\tau \gg 0$, and we conclude that $\phi$ is continuous on $\left[B_{1}, B\right] \subset\left[B_{1}, B_{2}\right)$.

Let us now prove that $\phi$ is a surjection in the whole interval $\left[t_{B_{2}}, t_{B_{1}}\right]$ if $u_{L}(t)+u_{R}(t)$ decreases at 0 . Let us consider $\tau \in\left[t_{B_{2}}, t_{B_{1}}\right]$ such that $\tau \simeq 0$. The existence of a solution $x(t)$ of $P_{M}$ with the behavior described above and, thus, the existence of a value $B \in\left[B_{1}, B_{2}\right]$ such that $\phi(B)=\tau$, may be deduced by continuity arguments as follows. Consider, in the $(t, x, V)$ space, a trajectory $\gamma(t)$ starting at a point $\left(0, A, V_{0}\right)$ in the vertical line $r_{A}, A=A^{*}$. If $V_{0} \ll 0$, the solution will satisfy $x(\tau)<M$. If $V_{0} \gg$ 0 , the solution $x(t)$ must satisfy $x(t)>x_{B_{2}}(t)$ because of the uniqueness of solutions of boundary-value problems, and so $x(\tau)>x_{B_{2}}(\tau)$. If $\tau$ is such that $x_{B_{2}}(\tau) \simeq u_{R}(\tau)$, we have $x(\tau)>M$. If, on the contrary, $\tau$ is such that $x_{B_{2}}(\tau) \ll u_{R}(\tau)$, the solution $x_{B_{2}}(t)$ increases in $\left[t_{B_{2}}, \tau\right]$ and as $u_{L}(t)+u_{R}(t)$ decreases for $t \simeq 0$, we also have $x(\tau)>M$. By continuity of the flow, there is $V_{0} \simeq 0$ for which $x(\tau)=M$, thus a solution of $P_{M}$ which satisfies $x(T)=B \in\left[B_{1}, B_{2}\right]$, and finally, we have the existence of $B$ such that $\phi(B)=\tau$.

Remark 4.2.3. Theorem 4.2.2 states that the shock location $t_{0}(A, B)$ is a continuous function of $B$ when $A=A^{*}$, and it moves towards 0 as $B \rightarrow B_{2}$ and towards $T$ as $B \rightarrow B_{1}$. Analogously, we can show that the shock layer $t_{0}$ depends continuously on $A$ when $B$ is fixed, $B=B^{*}$, and it moves towards 0 as $A \rightarrow A_{2}$ and towards $T$ as $A \rightarrow A_{1}$. Note that though the transition between a solution with a boundary layer at $t_{0}=0$ and one with a boundary layer at $t_{0}=T$ is continuous, it is not $S$-continuous at $B=B^{*}$ since $B_{1} \simeq B_{2}$ but $\phi\left(B_{1}\right) \not \phi\left(B_{2}\right)$.

The function $\phi$ itself has a jump at $B^{*}$ of extremities 0 and $T$. The thickness of this jump is given by $\xi=\{B: 0 \ll \phi(B) \ll T\}$, and it characterises the sensitivity of the boundary-value problem $P(A, B)$, i.e., the set of values $B \simeq B^{*}$ for which there are internal layers.

\section{Some examples}

Example 5.1. Consider the quasilinear problem

$$
\begin{aligned}
& \varepsilon x^{\prime \prime}=-2 x x^{\prime}+e^{t} \quad 0<t<1, \\
& x(0)=A, \quad x(1)=B .
\end{aligned}
$$

In the slow surface $S$, the reduced equation $-2 u u^{\prime}+e^{t}=0$ will be satisfied, so integration implies that we must have $u^{2}=e^{t}+C$ for some constant $C$. Since ${ }^{0} f_{v}\left(t, u, u^{\prime}\right)=-2 u$, those "reduced" trajectories $\left(t, u, u^{\prime}\right)$ with $u<0$ move in $S_{R}$ while, for $u>0$, they move in $S_{A}$. 
Consider the reduced solutions $u_{L}(t)$ and $u_{R}(t)$ determined by the boundary conditions $u_{L}(0)={ }^{0} A$ and $u_{R}(1)={ }^{0} B$ with $A$ and $B$ limited values.

In the interval $[0,1]$, any jump from $u_{L}(t)$ is described, in the observability space, by $G(t, x)=-x^{2}+u_{L}^{2}(t)$. When the boundary conditions are $A^{*}={ }^{0} A$ with $A<0$ and $B^{*}=\sqrt{A^{*^{2}}-1+e}$, we have $u_{R}(t)=-u_{L}(t)$ and the Rankine-Hugoniot condition $J(t)=G\left(t, u_{R}(t)\right) \equiv 0$ is satisfied in the whole interval [0,1]. In addition, the surface $S^{*}$ containing the jumps satisfies $G(t, x) \gg 0$ at any $t \in[0,1]$ and $u_{R}(t) \ll x \ll u_{L}(t)$ and $G_{X}=-2 x$ is positive for $x \leq u_{L}(t)$ and negative when $x \geq u_{R}(t)$. Then, Theorem 4.2.1 implies that this problem is supersensitive with respect to $\left(A^{*}, B^{*}\right)$. Thus, for any standard $t_{0} \in[0,1]$, there are $A \simeq A^{*}, B \simeq B^{*}$ and a solution $x(t)$ of the problem that jumps at $t_{0}$ and satisfies

$$
x(t) \simeq\left\{\begin{array}{ll}
u_{L}(t) & 0 \leq t \leq t_{L} \\
u_{R}(t) & t_{R} \leq t \leq 1
\end{array} \quad \text { with }{ }^{0} t_{L}={ }^{0} t_{R}=t_{0} .\right.
$$

Using Lorenz' results [17] concerning the uniqueness of solutions of boundary problems of the form

$$
\begin{gathered}
-\varepsilon x^{\prime \prime}+\alpha(t, x) x^{\prime}+\beta(t, x)=0 \quad 0<t<1, \\
x(0)=A, \quad x(1)=B,
\end{gathered}
$$

we remark that our two-point problem has a unique solution for any prescribed boundary values since, in this case, Lorenz's condition $\left(\beta_{x}-\alpha_{t}\right)(t, x) \geq 0$ holds for all $(t, x) \in[0,1] \times \mathbb{R}$. Finally, $u_{R}(t)+u_{L}(t) \equiv 0$, so we can apply Theorem 4.2.2 to conclude that the location of the shock $t_{0}$ depends continuously on the boundary conditions. The transition between a solution with a boundary layer at 0 and one with a boundary layer at 1 takes place in some infinitesimal interval $\left[B_{1}, B_{2}\right]$ with $B_{1} \simeq B_{2} \simeq B^{*}$ when $A$ is fixed. The shock layer moves to the right as $B \rightarrow B_{2}$ and to the left as $B \rightarrow B_{1}$.

\section{Example 5.2.}

$$
\begin{aligned}
& \varepsilon x^{\prime \prime}=-2 x x^{\prime}+1-x^{2} \quad 0<t<1 \\
& x(0)=A, \quad x(1)=B .
\end{aligned}
$$

In the slow surface $S$ of equation $-2 x v+1-x^{2}=0$, the reduced solutions are given by $u \equiv-1, u \equiv 1$, and $\left|1-u^{2}\right|=c e^{-t}, c>0$. The attractive region $S_{A}$ is obtained for those points of $S$ where $x>0$ and the repulsive region $S_{R}$ for those where $x<0$. Any jump from a reduced solution $u(t)$ is contained, in the observability space, in the corresponding surface of equation $V(t, x)=-x^{2}+u^{2}$. Then, the Rankine-Hugoniot condition is satisfied in $[0,1]$ for any couple of reduced solutions $\left(u_{L}(t), u_{R}(t)\right)$ with $u_{R} \equiv-u_{L}$. Therefore, it is easy to verify that hypothesis $\mathrm{H}$ is satisfied for the following set of boundary conditions $A^{*}={ }^{0} A$ and $B^{*}=\sqrt{1-\left(1-A^{* 2}\right) e^{-1}}$ for $-1 \leq A<0$ and $A^{*}={ }^{0} A$ and $B^{*}=\sqrt{1+\left(A^{*^{2}}-1\right) e^{-1}}$ for $A<-1$. Then, it follows from Theorem 4.2.1 that this problem exhibits the phenomena of supersensitivity with respect to each set of boundary values $\left(A^{*}, B^{*}\right)$; see Figure 5.1 .

Example 5.3. Consider the following quadratically nonlinear problem:

$$
\begin{aligned}
& \varepsilon x^{\prime \prime}=-2 x x^{\prime[2]}+2 t \quad 0<t<1, \\
& x(0)=A, \quad x(1)=B
\end{aligned}
$$

where $x^{\prime[2]}=x^{\prime}\left|x^{\prime}\right|$.

The slow surface $S$ is given by $-x v^{[2]}+t=0$ for $x \neq 0$. Since $\partial^{0} f / \partial v=-4 x|v|$, any point of $S$ with $x<0$ belongs to the repulsive region while those with $x>0$ 

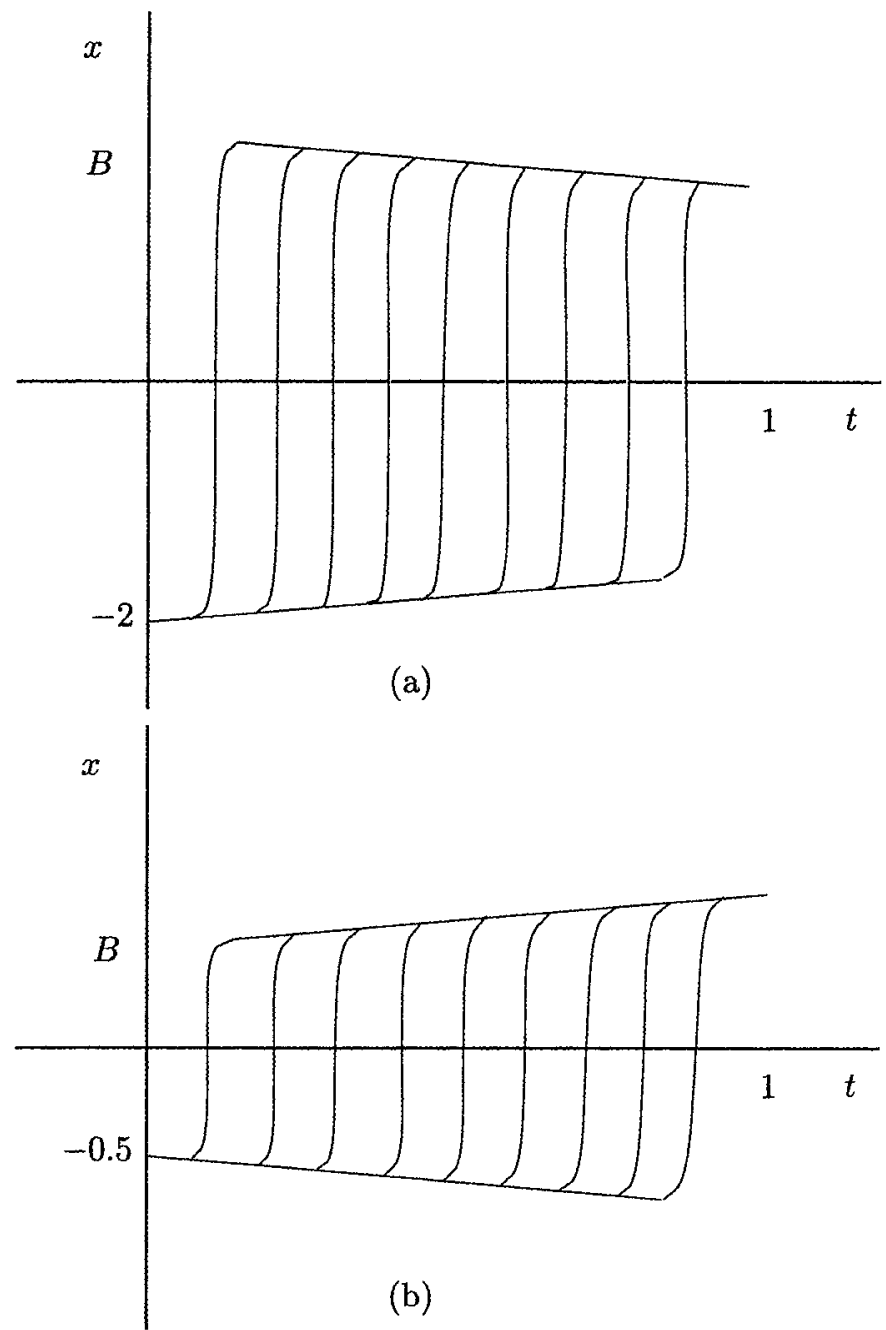

Figure 5.1 Solutions of $\varepsilon x^{\prime \prime}=-2 x x^{\prime}+1-x^{2}$ with $\varepsilon=0.01$ and

(a) $x(0)=-2$ and $x(1)=B$ for nine values of $B$ close to $\sqrt{1+3 e^{-1}}$,

(b) $x(0)=-0.5$ and $x(1)=B$ for nine values of $B$ close to $\sqrt{1-0.75 e^{-1}}$.

belong to the attractive region. The reduced problem $u u^{\prime[2]}=t$ has, for $t>0$, the solutions $u= \pm\left(t^{3 / 2}+C\right)^{2 / 3}$. A similar analysis to that performed in the previous examples shows that the problem is supersensitive with respect to $A^{*}={ }^{0} A$ for any limited $A<0$ and $B^{*}=\left(1+\left(-A^{*}\right)^{3 / 2}\right)^{2 / 3}$. Thus for any $t_{0} \in[0,1]$, there is a solution of $P(A, B)$ with $A \simeq A^{*}, B \simeq B^{*}$ which moves infinitely close to $u_{L}(t)=$ $-\left(t^{3 / 2}+\left(-A^{*}\right)^{3 / 2}\right)^{2 / 3}$ until some time $t \simeq t_{0}$, where it jumps to the other reduced solution $u_{R}(t)=\left(t^{3 / 2}+\left(-A^{*}\right)^{3 / 2}\right)^{2 / 3}$, and then it spends the remaining time near $u_{R}(t)$.

Example 5.4. In [1] we have illustrated the phenomenon of supersensitivity with

$$
\begin{aligned}
& \varepsilon x^{\prime \prime}=\left((\gamma+1) / 2-x^{-2}\right) x^{\prime} \quad 0<t<1, \\
& x(0)=A, \quad x(1)=B .
\end{aligned}
$$


This problem arises in the study of compressible fluids in gas dynamics when a gas is injected at a supersonic velocity $A$ in a duct of uniform cross-sectional area and a back pressure is applied. Here $\gamma$ is the adiabatic index, $x$ is the dimensionless velocity of the gas relative to the the velocity of the sound, and $t$ is the dimensionless distance with $t=0$ at the entrance of the duct. The dimensionless constant $\varepsilon=\mu \gamma / \rho_{0} c_{0}$ is small when the coefficient of viscosity $\mu$ is small with respect to fixed values of $\gamma, \rho_{0}$, and $c_{0}$. The quantities $\rho_{0}$ and $c_{0}$ are, respectively, a reference density and the velocity of sound.

The physical problem may be stated as follows. Determine the subsonic velocity $B$ at $t=1$ that produces a supersonic-subsonic shock in the duct when a supersonic velocity $A$ is given at $t=0$ and also the location of the shock.

Any reduced solution $u$ is constant, and any jump from $u \equiv A$ is, in the observability space, infinitely close to the surface defined by

$$
G(t, x)=\left(\frac{\gamma+1}{2} x+\frac{1}{x}\right)-\left(\frac{\gamma+1}{2} A+\frac{1}{A}\right) .
$$

Then the Rankine-Hugoniot shock condition

$$
\left(\frac{\gamma+1}{2} B+\frac{1}{B}\right)-\left(\frac{\gamma+1}{2} A+\frac{1}{A}\right)=0
$$

holds in $[0,1]$ for the coupled boundary conditions which satisfy the well-known Prandtl relation

$$
A B^{*}=\frac{2}{\gamma+1},
$$

and for these values, there is a shock layer located at $t_{0}^{*}=A /\left(A+B^{*}\right)$, as we have shown in [1].

Since this is a supersensitive boundary-value problem with respect to $\left(A, B^{*}\right)$, we have that, for any $t_{0}$ in the interior of the duct, there is a solution of $P(A, B)$, which describes a supersonic-subsonic transition at $t_{0}$ between the constant states $u \equiv A$ and $u \equiv B^{*}$, with values of $B \simeq B^{*}$. In addition, because of the uniqueness of solutions of this boundary-value problem, the shock location $t_{0}$ moves continuously with $B$. It moves to the end of the duct for $B \geqq B^{*}$ as the quantity $B-B^{*}$ increases and to the entrance of the duct for $B \leqq B^{*}$ as $\bar{B}-B^{*}$ decreases.

The reasoning employed in this example extends to the family of problems of the form

$$
\begin{aligned}
& \varepsilon x^{\prime \prime}=\left((\gamma+1) / 2-x^{-2}\right) x^{[s]}+G(t, x) \quad 0<t<1, \\
& x(0)=A, \quad x(1)=B
\end{aligned}
$$

where $s$ is a real number, $1 \leq s \leq 2$.

This family is then another example of supersensitive boundary-value problem with respect to $\left(A, B^{*}\right)$ provided that the boundary values satisfy the Prandtl relation.

In [1], we have considered equations of the form $\varepsilon x^{\prime \prime}=g(x) f\left(x^{\prime}\right)$, and we have shown that the location of the internal layers can be perturbed significantly as a result of exponentially small changes in the boundary conditions when $f$ is a linear function. The boundary-value problem for Burgers' equation and the model for compressible fluid flow in nozzles are two examples of problems with exponential sensitivity.

On the contrary, the internal layer positions are sensitive to algebraically, rather than exponentially, small perturbations if $f\left(x^{\prime}\right)=O\left(x^{\prime s}\right)$ as $\left|x^{\prime}\right| \rightarrow+\infty$, with $1 \leq s \leq$ 2. More precisely, in this case, we have shown in [1] that the sensitivity is given by perturbations of order $O\left(\varepsilon^{3-s}\right)$. 


\section{References}

1. A. Bohe, Free layers in a singularly perturbed boundary value problem, SIAM J. Math. Anal. 21 (1990), 1264-1280.

2. Sauts singuliers dans des problèmes de perturbation singulière d'équations différentielles ordinaires, Thèse, Univ. Paris 7, 1991.

3. Yu. P. Boglaev, Smoothing of the solution in a control problem, USSR Comput. Math. and Math. Phys. 10 (1970), 226-230.

4. J. M. Burgers, A mathematical model illustrating the theory of turbulence, Adv. Appl. Mech. 1, 1948.

5. V. F. Butuzov and A. B. Vasil'eva, The asymptotic theory of contrasting spatial structures, USSR Comput. Math. and Math. Phys. 28 (1988), 26-36.

6. K. Chang and F. Howes, Nonlinear Singular Perturbation Phenomena: Theory and Application, Springer-Verlag, New York, 1984.

7. J. D. Cole, Perturbation Methods in Applied Mathematics, Blaisdell, Waltham, 1968.

8. L. Crocco, A suggestion for the numerical solution of the steady Navier-Stokes equations, AIAA J. 3 (1965), 1824-1832.

9. F. Diener, Méthode du plan d'observabilité, Thèse, Univ. Louis Pasteur, Strasbourg, 1981.

10. $\_$, Sauts des solutions des Equations $\varepsilon x^{\prime \prime}=f\left(t, x, x^{\prime}\right)$, SIAM J. Math. Anal. 17 (1986), 533-559.

11. __ Equations Surquadratiques et Disparition des sauts, SIAM J. Math. Anal. 19 (1988), 1127-1134.

12. F. Diener and G. Reeb, Analyse Non Standard, Hermann Eds., Paris, 1989.

13. P. C. Fife, Dynamics of Internal Layers and Diffusive Interfaces, SIAM, Philadelphia, 1988.

14. F. Howes, Boundary-interior layer interactions in nonlinear singular perturbation theory, Mem. Amer. Math. Soc. 203 (1978), 1-108.

15. J Kevorkian and J. D. Cole, Perturbations Methods in Applied Mathematics, Springer-Verlag, New York, 1981.

16. P. A. Lagerstrom, Matched Asymptotic Expansions, Springer-Verlag, New York, 1988.

17. J. Lorenz, Stability and monotonicity properties of stiff quasilinear boundary problems, Review of Research Faculty of Science-University of Novi Sad 12 (1982), 151-175.

18. R. Lutz and M. Goze, Non Standard Analysis. A Practical Guide with Applications, Lecture Notes in Math. 881, Springer-Verlag, New York, 1981.

19. D. W. McLaughlin, Path integrals, asymptotics and singular perturbations, J. Math. Phys. 13 (1972), 786-796.

20. B. J. Matkowsky, Singular perturbations, stochastic differential equations, and applications, in: Singular Perturbations and Asymptotics, (Eds. R. E. Meyer and S. V. Parter), Academic Press, New York, 1980, pp. 109-147.

21. R. E. O'Malley, Jr., Introduction to Singular Perturbations, Academic Press, New-York, 1974.

22. __ Singular Perturbation Methods for Ordinary Differential Equations, Springer-Verlag, New York, 1991.

23. - Singular perturbations, asymptotic evaluation of integrals, and computational challenges, in: Asymptotic Analysis and the Numerical Solution of Partial Differential Equations, (Eds. Hans G. Kaper and Marc Garbey), Marcel Dekker, New York, 1991.

24. I. P. van den Berg, Non Standard Analysis, Lectures Notes in Mathematics, 1249, Springer-Verlag, 1987.

25. M. Visik and L. A. Liusternik, Initial jump for nonlinear differential equations containing a small parameter, Soviet Math. Dokl. 1 (1960), 749-752.

Université Paris 7, 2, Place Jussieu, 75251 Paris Cedex O5, France 